\title{
Recent Progress in NEO-2 - A Code for Neoclassical Transport Computations Based on Field Line Tracing
}

\author{
Winfried KERNBICHLER ${ }^{1)}$, Sergei V. KASILOV ${ }^{1,2)}$, Georg O. LEITOLD ${ }^{1)}$, \\ Viktor V. NEMOV ${ }^{1,2)}$ and Klaus ALLMAIER ${ }^{1)}$ \\ ${ }^{1)}$ Association EURATOM-ÖAW, Institut für Theoretische Physik - Computational Physics, \\ TU Graz, Petersgasse 16, A-8010 Graz, Austria \\ ${ }^{2)}$ Institute of Plasma Physics, National Science Center "Kharkov Institute of Physics and Technology", \\ Akademicheskaya Str. 1, 61108 Kharkov, Ukraine
}

(Received 16 November 2007 / Accepted 13 February 2008)

\begin{abstract}
NEO-2 is a code for the computation of neoclassical transport coefficients and current drive efficiency in toroidal devices which is based on the field line integration technique. The possibility to use the complete linearized collision integral is realized in this code. In this report the results of comparison of the full matrix of transport coefficients in a tokamak with analytical models are presented. Effects of simplifications of the linearized collision model (e.g., reduction to a Lorentz model) are studied in order to provide a comparison with various momentum correction techniques used for the computation of transport coefficients in stellarators.
\end{abstract}

(c) 2008 The Japan Society of Plasma Science and Nuclear Fusion Research

Keywords: neoclassical transport, linearized collision operator

DOI: $10.1585 /$ pfr.3.S1061

\section{Introduction}

Accurate computations of transport coefficients, bootstrap current and the generalized Spitzer function in tokamaks and stellarators is an important problem for stellarator optimization, generation of neoclassical data bases, and modeling of current drive. Based on the field line integration technique [1], the code NEO-2 has been developed for this purpose [2,3]. This code solves the linearized drift kinetic equation in regimes where the effect of electric field on transport and bootstrap coefficients is negligible. Recently this code has been upgraded for computations of the full transport matrix and the possibility of treatment of magnetic fields in Boozer coordinates has been realized in addition to magnetic fields in real space coordinates. In the following, a comparison of NEO-2 results with results of analytical theory for tokamak are presented. Transport coefficients are computed here only for the electron component assuming the ions to be immobile.

\section{Basic Equations}

Approximating the energy dependence of the perturbation of the distribution function by the expansion over a finite number of test functions,

$$
\begin{aligned}
& \delta f(\psi, s, v, \lambda) \approx f_{0}(\psi, v) \sum_{m=0}^{M} f_{m}(\psi, s, \lambda) \varphi_{m}\left(v / v_{T}\right), \\
& \varphi_{m}(x)=\pi^{3 / 4} \sqrt{\frac{2 \Gamma(m+1)}{\Gamma(m+5 / 2)}} \mathrm{L}_{m}^{(3 / 2)}\left(x^{2}\right),
\end{aligned}
$$

author's e-mail: winfried.kernbichler@TUGraz.at where $f_{0}(\psi, v)$ is the Maxwellian and $\mathrm{L}_{m}^{(3 / 2)}$ are associated Laguerre polynomials (Sonine polynomials) of the order $3 / 2$, the linearized drift kinetic equation (DKE) is transformed to a set of coupled two dimensional differential equations,

$$
\begin{aligned}
\sigma \frac{\partial f_{m}^{\sigma}}{\partial s} & -\kappa \sum_{m^{\prime}=0}^{M}\left\{v_{m m^{\prime}} \mathcal{L} f_{m^{\prime}}^{\sigma}+\mathcal{K}_{m m^{\prime}} f_{m^{\prime}}^{\sigma}+\frac{1}{|\lambda|} D_{m m^{\prime}} f_{m^{\prime}}^{\sigma}\right\} \\
& =\frac{\rho}{\langle|\nabla \psi|\rangle}\left(A_{1} a_{m}^{(1)}+A_{2} a_{m}^{(2)}\right) q_{G}^{\sigma}+A_{3} a_{m}^{(3)} q_{E}^{\sigma}
\end{aligned}
$$

with the pitch-angle scattering operator

$$
\begin{aligned}
\mathcal{L} f_{m^{\prime}}^{\sigma} & =\frac{1}{2|\lambda|} \frac{\partial}{\partial \lambda}\left(1-\lambda^{2}\right) \frac{\partial}{\partial \lambda} f_{m^{\prime}}^{\sigma}(\psi, s, \lambda) \\
& =2 \frac{\partial}{\partial \eta}\left(\frac{\eta|\lambda|}{\hat{B}}\right) \frac{\partial}{\partial \eta} f_{m^{\prime}}^{\sigma}(\psi, s, \eta),
\end{aligned}
$$

and the integral part of the linearized collision operator

$\mathcal{K}_{m m^{\prime}} f_{m^{\prime}}^{\sigma}=\frac{1}{|\lambda|} \sum_{\ell=0}^{L} I_{m m^{\prime}}^{\ell} \mathrm{P}_{\ell}(\lambda) \int_{-1}^{1} \mathrm{~d} \lambda^{\prime} \mathrm{P}_{\ell}\left(\lambda^{\prime}\right) f_{m^{\prime}}^{\sigma^{\prime}}\left(\psi, s, \lambda^{\prime}\right)$,

where $\mathrm{P}_{\ell}$ are Legendre polynomials. Here, $\psi$ is a flux surface label, $s$ is the distance counted along the magnetic field line, $\lambda=v_{\|} / v$ is pitch, $\sigma$ is the sign of $v_{\|}, \eta=$ $\left(1-\lambda^{2}\right) / \hat{B}$ is the normalized perpendicular invariant (magnetic moment), $\hat{B}=B / B_{0}$ is the magnetic field module normalized to a reference magnetic field $B_{0}$ which is equal to $(0,0)$ harmonic of magnetic field expansion in Boozer coordinates, $B_{0}=\left\langle B^{3}\right\rangle /\left\langle B^{2}\right\rangle, \kappa=2 / l_{\mathrm{c}}$ with $l_{\mathrm{c}}=v_{T} \tau_{\mathrm{ee}}$ being the mean free path, $\rho=v_{T} / \omega_{\mathrm{c}}, v_{T}=\left(2 T_{\mathrm{e}} / m_{\mathrm{e}}\right)^{1 / 2}$, 
$\omega_{\mathrm{c}}=e B_{0} / m_{\mathrm{e}} c$ and $\tau_{\mathrm{ee}}=3 m_{\mathrm{e}}^{2} v_{T}^{3} /\left(16 \sqrt{\pi} n_{\mathrm{e}} e^{4} \Lambda\right)$ are electron Larmor radius, thermal velocity, cyclotron frequency and collision time, respectively, and $\langle\ldots\rangle$ means the average over the volume between two neighboring magnetic surfaces (flux surface average). The quantities $v_{m m^{\prime}}, I_{m m^{\prime}}$, $D_{m m^{\prime}}$ and $a_{m}^{(i)}$ are matrix elements independent of plasma parameters, whereas the quantities $A_{i}$ are the thermodynamic forces defined as,

$$
\begin{aligned}
& A_{1}=\left(\frac{1}{n} \frac{\partial n}{\partial \psi}-\frac{3}{2 T} \frac{\partial T}{\partial \psi}+\frac{e}{T} \frac{\partial \Phi}{\partial \psi}\right)\langle|\nabla \psi|\rangle, \\
& A_{2}=\frac{1}{T} \frac{\partial T}{\partial \psi}\langle|\nabla \psi|\rangle, \quad A_{3}=\frac{e}{T} \frac{\left\langle E_{\|} \hat{B}\right\rangle}{\left\langle\hat{B}^{2}\right\rangle} .
\end{aligned}
$$

The source terms $q_{G}^{\sigma}$ and $q_{E}^{\sigma}$ with drives by gradients and by parallel electric field, respectively, are defined as follows,

$$
\begin{aligned}
& q_{G}^{\sigma}=\frac{\partial}{\partial \eta}\left(\frac{|\lambda|}{\hat{B}} \hat{V}_{G}\right), \quad q_{E}^{\sigma}=\sigma \hat{B}, \\
& \hat{V}_{G}=\frac{1}{3}\left(\frac{4}{\hat{B}}-\eta\right)|\nabla \psi| k_{G},
\end{aligned}
$$

where $k_{G}$ is the geodesic curvature.

Equations (2) are discretized over the $\eta$ variable on an adaptive non-equidistant grid and the resulting set of coupled ordinary differential equations is solved with help of integration along the field lines. In particular, all necessary flux surface averages are computed during this integration as follows,

$$
\frac{\langle\alpha\rangle}{\langle\beta\rangle}=\lim _{L \rightarrow \infty} \int_{0}^{L} \frac{\mathrm{d} s}{B} \alpha / \int_{0}^{L} \frac{\mathrm{d} s}{B} \beta .
$$

As a result of computation, one obtains the matrix of transport coefficients $L_{i j}^{\mathrm{e}}$ defined through the thermodynamic forces $A_{j}$ and thermodynamic fluxes $I_{i}$ via

$$
I_{i}=-\sum_{j=1}^{3} L_{i j}^{\mathrm{e}} A_{j}
$$

where $I_{1}=\Gamma_{\mathrm{e}}, I_{2}=Q_{\mathrm{e}} / T_{\mathrm{e}}$ and $I_{3}=\left\langle j_{\|} \hat{B}\right\rangle / e$. Here $\Gamma_{\mathrm{e}}$ and $Q_{\mathrm{e}}$ are average particle and heat flux densities defined as total particle and heat fluxes divided by the flux surface area and $j_{\|}$is parallel electron current density.

Symmetric matrix $L_{i j}^{\mathrm{e}}$ is conveniently expressed through dimensionless coefficients $\gamma_{i j}$ which depend only on the device geometry, the mean free path $l_{\mathrm{c}}$ and effective charge number $Z$ as follows, $L_{i j}=n_{\mathrm{e}} \gamma_{i j} \beta_{i} \beta_{j} / \tau_{\mathrm{ee}}$, where $\beta_{1}=\beta_{2}=\rho$ and $\beta_{3}=l_{\mathrm{c}}$. For this purpose solution to (2) is formally presented as a superposition of thermodynamic forces,

$$
f_{m}^{\sigma}=\frac{\rho}{\langle|\nabla \psi|\rangle}\left(A_{1} \hat{f}_{m}^{\sigma,(1)}+A_{2} \hat{f}_{m}^{\sigma,(2)}\right)+A_{3} \hat{f}_{m}^{\sigma,(3)} .
$$

Then $\gamma_{i j}$ are determined by normalized solutions for single drive problems, $\hat{f}_{m}^{\sigma,(i)}$, as follows

$$
\gamma_{i j}=\frac{\alpha_{i} \alpha_{j}}{l_{\mathrm{c}}} \sum_{m} \sum_{\sigma= \pm 1} b_{m}^{(i)}\left\langle\hat{B} \int_{0}^{1 / \hat{B}} \mathrm{~d} \eta \hat{f}_{m}^{\sigma,(j)} q_{i}^{-\sigma}\right\rangle,
$$

where $q_{1}^{\sigma}=q_{2}^{\sigma}=q_{G}^{\sigma}, \quad q_{3}^{\sigma}=q_{E}^{\sigma}, \quad \alpha_{1}=\alpha_{2}=l_{\mathrm{c}}\langle|\nabla \psi|\rangle^{-1}$, $\alpha_{3}=1$ and numerical coefficients $b_{m}^{(i)}$ are, again, independent of problem parameters. With help of these coefficients the quantity $\lambda_{b b}$ [3] is expressed as $\lambda_{b b}=2 \gamma_{31}$.

It should be noted that matrices $L_{i j}^{\mathrm{e}}$ and $\gamma_{i j}$ correspond to the effective radius $r_{\text {eff }}$ used as a radial variable where $\mathrm{d} r_{\text {eff }}=\mathrm{d} V / S, V$ is a volume limited by a flux surface and $S$ is a flux surface area. In order to obtain these matrices for different definitions of plasma radius, e.g. for the radius defined via the toroidal flux, $r_{\psi}=\left(2 \psi / B_{00}\right)^{1 / 2}$, coefficients $\alpha_{1}$ and $\alpha_{2}$ should be multiplied with $\mathrm{d} r_{\psi} / \mathrm{d} r_{\text {eff }}$.

\section{Computational Results}

For the large aspect ratio tokamak with circular flux surfaces coefficients $\gamma_{i j}$ computed by NEO-2 are compared to the analytical results of Refs. [4-6]. In particular, dimensionless transport coefficients for the Hinton and Hazeltine model [4], $\gamma_{i j}^{\mathrm{HH}}$, are given by $\gamma_{i j}^{\mathrm{HH}}=$ $K_{i j} q^{2} \epsilon_{\mathrm{t}}^{-3 / 2} Z_{\mathrm{eff}}$ for $i, j=1,2, \gamma_{i j}^{\mathrm{HH}}=K_{i j} q \epsilon_{\mathrm{t}}^{-1 / 2} / 2$ for $i=1,2$ and $j=3$ or $i=3$ and $j=1,2$,

$$
\gamma_{33}^{\mathrm{HH}}=\frac{K_{33} \epsilon_{\mathrm{t}}^{1 / 2}-1}{2 Z_{\mathrm{eff}}\left(0.29+0.46\left(1.08+Z_{\mathrm{eff}}\right)^{-1}\right)},
$$

and matrix $K_{i j}$ is defined by Eqs. (6.125) and (6.126) of Ref. [4]. Here $q$ is safety factor and $\epsilon_{\mathrm{t}}=r / R$ is the inverse aspect ratio. The results of the comparison are presented in Figs. 1 and 2 for $t=1 / q=0.362$ and $\epsilon_{\mathrm{t}}=0.075$. The results of NEO-2 are computed with associated Laguerre polynomials up to fourth order and Legendre polynomials up to third order. For all coefficients the dependence on collisionality as well as $Z_{\text {eff }}$ is well reproduced. The main differences come from the finite toroidicity. Whereas NEO-2 does not assume smallness of the magnetic field modulation, theoretical approximations are based on the expansion over $\epsilon_{\mathrm{t}}$. It should be noted that in NEO-2 all nine transport coefficients are computed independently and Onsager symmetry of these coefficients is used for the control of the computation accuracy which improves with both, grid resolution and (mainly) number of Laguerre polynomials in modeling energy dependence. For the present computation violation of symmetries $\gamma_{13}=\gamma_{31}$ and $\gamma_{23}=$ $\gamma_{32}$ is around $1 \%$ and violation of symmetry $\gamma_{12}=\gamma_{21}$ is around $10 \%$.

Beside the full linearized collision operator, two different model operators are used in Fig. 3, namely, the mono-energetic collision model and mono-energetic collision model with momentum recovery. Last two models are obtained by putting in (2) $D_{m m^{\prime}}=I_{m m^{\prime}}^{\ell}=0$ or only $D_{m m^{\prime}}=0$, respectively. In particular, the monoenergetic model here corresponds to the most common mono-energetic approach where transport coefficients are given by the convolution over energy of the results for the Lorentz model. It can be seen that mono-energetic model overestimates particle diffusion coefficient $\gamma_{11}$ while mono-energetic model with momentum recovery underes- 


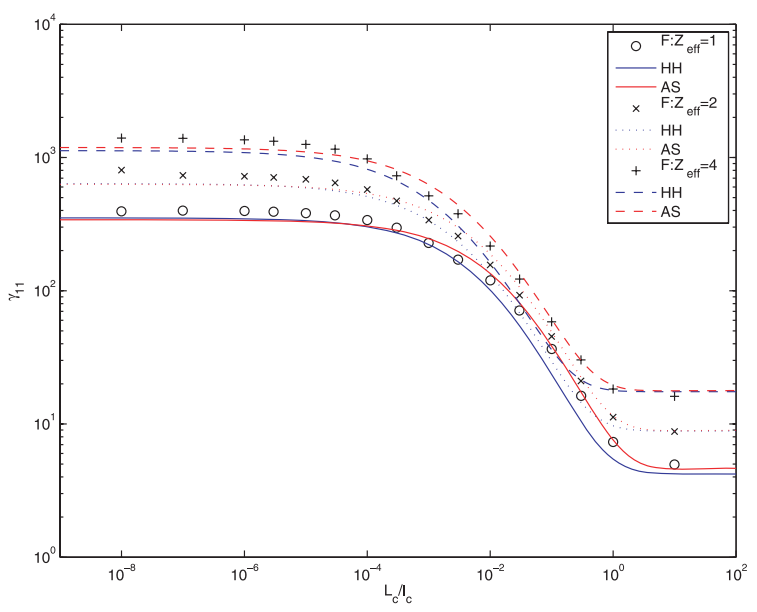

(a)

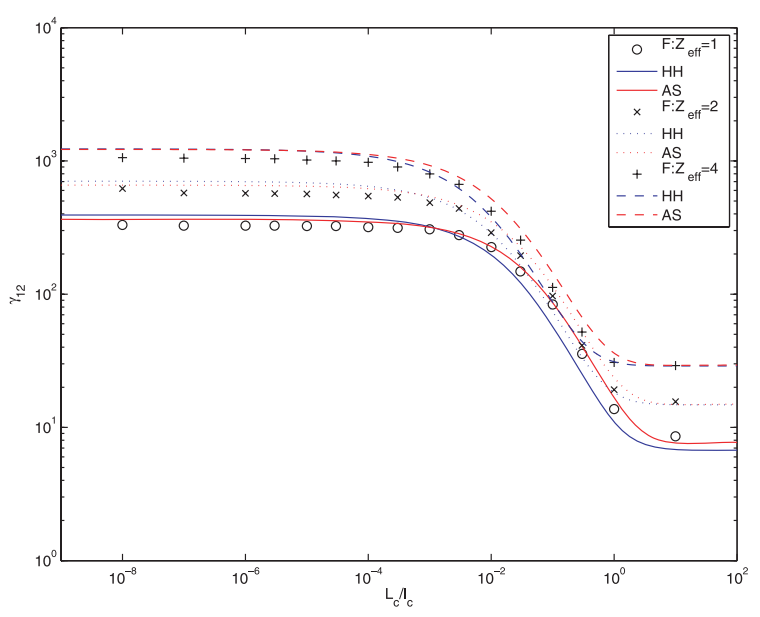

(b)

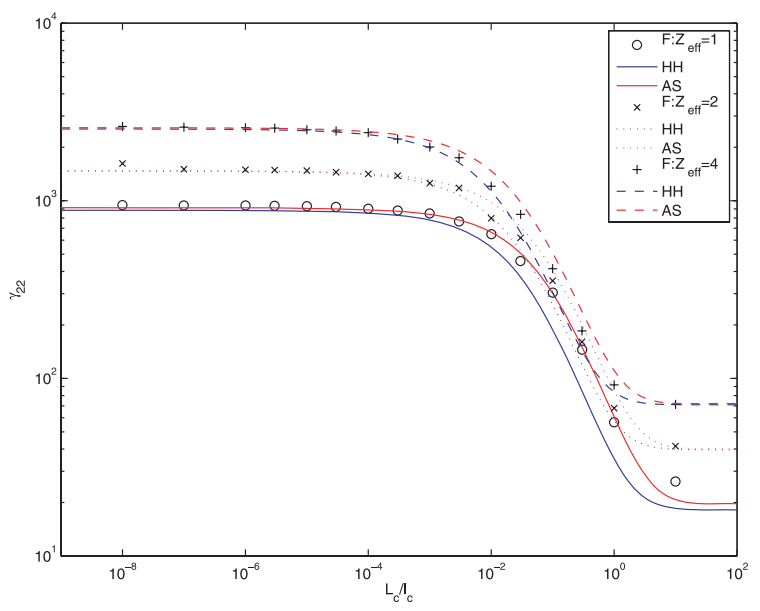

(c)

Fig. 1 Results of NEO-2 with full linearized collision operator (F) and analytical models of Ref. [4] (HH) and Refs. [5,6] (AS) for the dimensionless transport coefficients $\gamma_{11}$ (a), $\gamma_{12}$ (b) and $\gamma_{22}$ (c), respectively, at three values of the effective charge $Z_{\text {eff }}$.

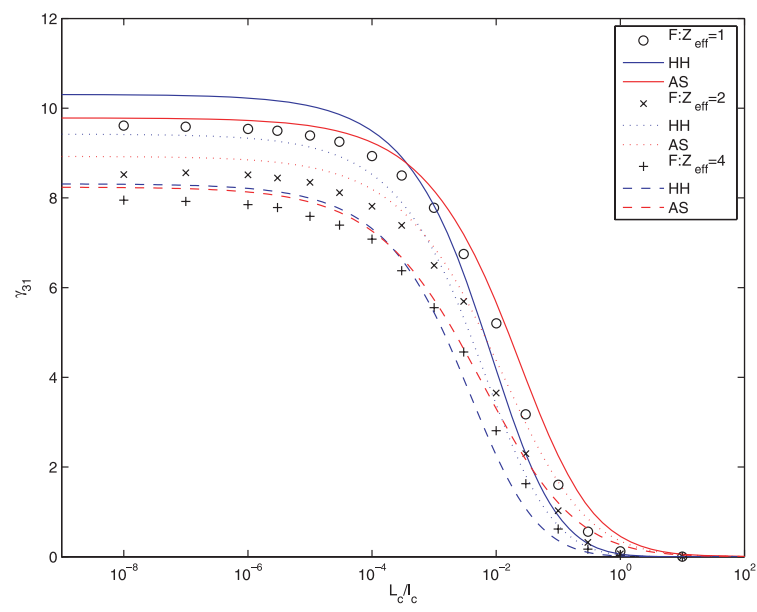

(a)

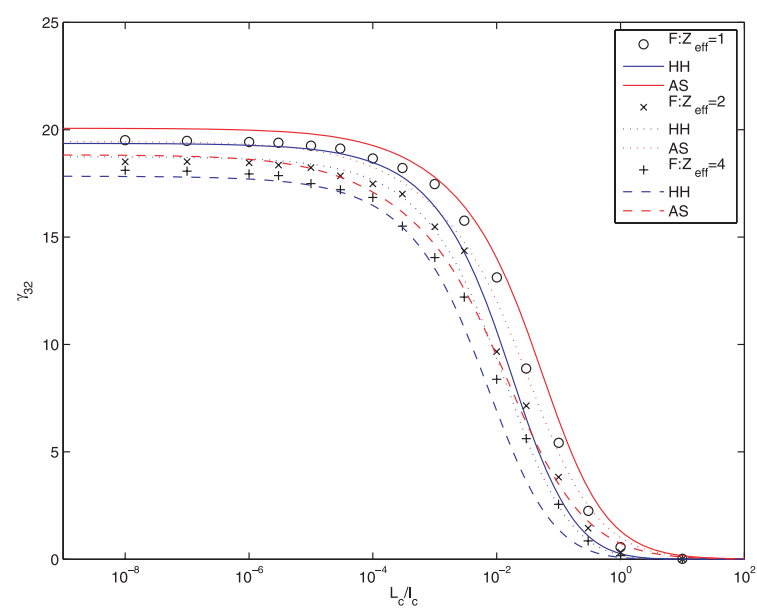

(b)

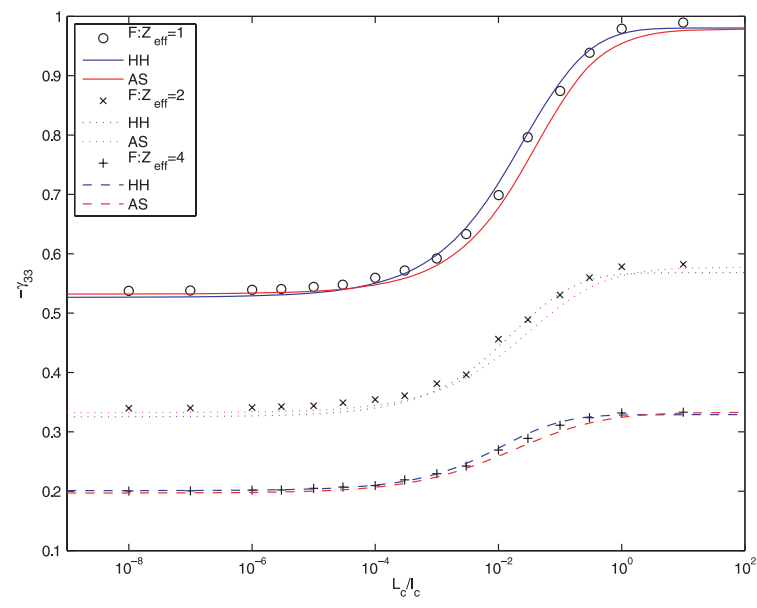

(c)

Fig. 2 The same as in Fig. 1 for the transport coefficients $\gamma_{31}$ (a), $\gamma_{32}$ (b) and $-\gamma_{33}$ (c), respectively. 


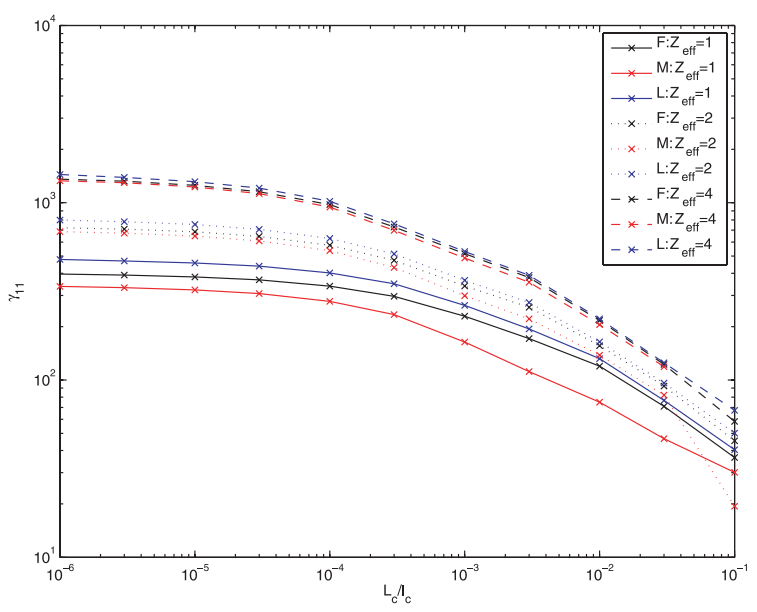

(a)

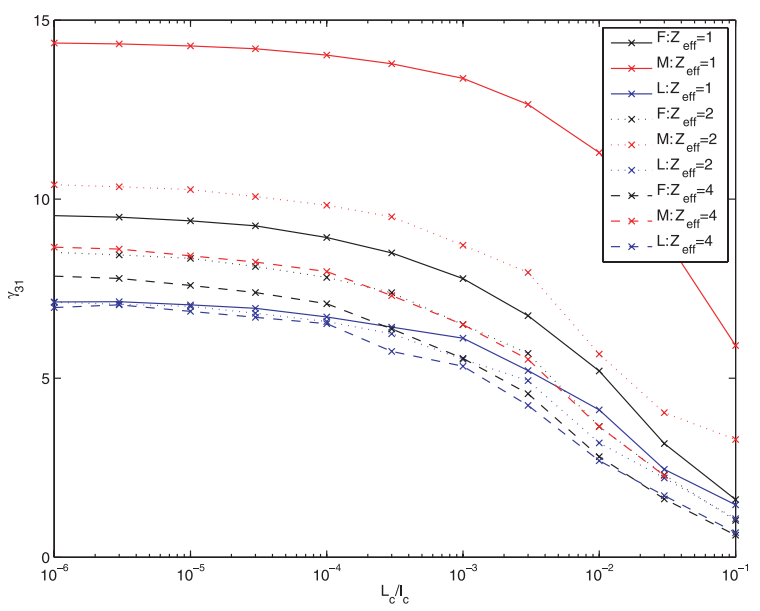

(b)

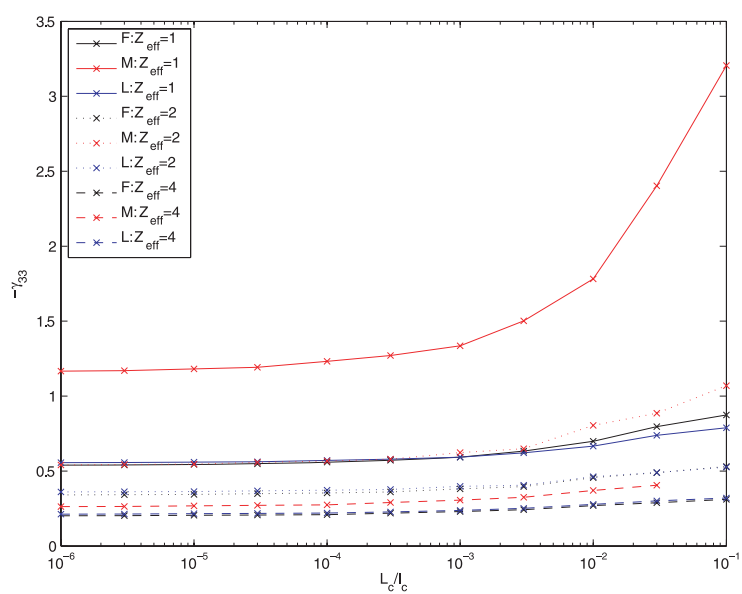

(c)

Fig. 3 Dimensionless particle diffusion coefficient $\gamma_{11}$ (a), the bootstrap coefficient $\gamma_{31}$ (b) and the conductivity coefficient $-\gamma_{33}$ (c), respectively, for the full linearized collision operator $(\mathrm{F})$, mono-energetic approach (L) and mono-energetic approach with momentum recovery $(\mathrm{M})$. timates this coefficient as compared to the full linearized collision model. The bootstrap coefficient $\gamma_{31}$, in turn, is underestimated by the mono-energetic model while the mono-energetic model with momentum recovery overestimates it. Finally, conductivity coefficient is well reproduced by the mono-energetic model while the monoenergetic model with momentum recovery significantly overestimates it. Differences between all three models are naturally reduced with higher $Z_{\text {eff }}$. Currently NEO-2 has been and is being used for the benchmarking of various methods for the computation of mono-energetic transport coefficients and bootstrap coefficient $[7,8]$ as well as momentum correction techniques [9].

\section{Acknowledgments}

This work, supported by the European Communities under the contract of Association between EURATOM and the Austrian Academy of Sciences, was carried out within the framework of the European Fusion Development Agreement. The views and opinions expressed herein do not necessarily reflect those of the European Commission. Additional funding is provided by the Austrian Science Foundation, FWF, under contract number P16797N08.

[1] V.V. Nemov, S.V. Kasilov, W. Kernbichler and M.F. Heyn, Phys. Plasmas 6, 4622 (1999).

[2] W. Kernbichler, S.V. Kasilov, G.O. Leitold, V.V. Nemov and K. Allmaier, Proc. of the 15th Int. Stellarator Workshop, Madrid, 3-7 October 2005, P2-15 (2005).

[3] W. Kernbichler, S.V. Kasilov, G.O. Leitold, V.V. Nemov and K. Allmaier, 33rd EPS Conference on Plasma Physics, Rome, 19-23 June 2006, ECA Vol. 30I P-2.189 (2006).

[4] F.L. Hinton and R.D. Hazeltine, Rev. Mod. Phys 48, 239 (1976).

[5] O. Sauter, C. Angioni and Y.R. Lin-Liu, Phys. Plasmas 6, 2834 (1999).

[6] C. Angioni and O. Sauter, Phys. Plasmas 7, 1224 (2000).

[7] K. Allmaier et al., Proc. of the 16th International Stellarator/Heliotron Workshop, Ceratopia Toki, Gifu, Japan, 15-19 October 2007, P2-029 (2007).

[8] K. Allmaier et al., Proc. of the 16th International Stellarator/Heliotron Workshop, Ceratopia Toki, Gifu, Japan, 15-19 October 2007, P2-021 (2007).

[9] C.D. Beidler et al., Proc. of the 16th International Stellarator/Heliotron Workshop, Ceratopia Toki, Gifu, Japan, 15-19 October 2007, P2-030 (2007). 